\title{
Multiple Atraumatic Osteoporotic Vertebral Fractures in an Adolescent with Suprasellar Germinoma: A Case Report
}

\author{
CC Wong, MD (USM), HF Phang, MS Orth (UKM), ML Cheong, M S Orth (UM), \\ Ramanathan Ramiah, FRCS \\ Department of Orthopaedics, Hospital Raja Permaisuri Bainun, Ipoh, Malaysia
}

\begin{abstract}
We present the case of a patient with multiple atraumatic osteoporotic vertebral fractures in an adolescent with suprasellar germinoma and also review of relevant literature. The patient suffered from a rare adolescent brain tumour with common complications which are often overlooked and give rise to significant morbidity. Suprasellar germinoma is an intracranial neoplasm, that in addition to its rarity, has variable clinical presentation. Despite appropriate treatment and good outcome, tumour related morbidity is still of concern for these patients.
\end{abstract}

\section{Key Words:}

Secondary osteoporosis, suprasellar germinoma, panhypopituitarism

\section{INTRODUCTION}

This report presents a case of suprasellar germinoma complicated with panhypopituitarism in an 18-year-old male. Despite treatment with hormonal replacement therapy, he presented to us with complaint of back pain of 6 months duration. The patient was diagnosed with secondary osteoporosis with multiple vertebral fractures.This case report, in addition to a review of the literature, shows that the pathogenesis of osteoporosis is multifactorial. Clinicians need to be aware of the clinical symptoms and complications of suprasellar germinoma with panhypopituitarism. Hormonal replacement alone is insufficient to prevent osteoporosis in these patients. In the present case, combination therapy was initiated and follow-up evaluation showed improvement.

Suprasellar germinoma is an intracranial neoplasm with peak incidence from 10-19 years of age ${ }^{1}$. Its clinical presentations typically consist of endocrine abnormalities and effects of the mass in the brain. Suprasellar germinomas are extremely radiosensitive tumours; thus combined with surgical resection, treatment can result in an excellent outcome. Panhypopituitarism is a cause of morbidity in these cases that can include development of osteoporosis, a sequelae of endocrine dysfunction which is often overlooked and under treated.
We present a case report of multiple atraumatic osteoporotic vertebral fractures in an adolescent with suprasellar germinoma. This rare condition is often overlooked and requires early attention to minimize complications.

\section{CASE REPORT}

An 18-year old boy presented with a history of panhypopituitarism diagnosed 7 years previously with a multiple resonance imaging (MRI) scan which was consistent with suprasellar germinoma. He was started on hormonal therapy (hydrocortisone, testosterone and thyroxine) at the time of diagnosis. Transphenoidal resection was done 1 year after diagnosis and followed by 30 cycles of radiotherapy. After 2 years of hormonal treatment, he presented to us with complaint of back pain for the previous 6 months, following which he had been using walking stick for ambulation. Otherwise, he had no history of trauma or fever. On examination, he appeared cachexic with tenderness over the thoracic spine, but with no neurological deficit. The remainder of clinical examination was unremarkable.

Thoracolumbar and lumbosacral spine radiographs showed T10, T12 and L1 compression fractures with osteopenic changes. A dual X-ray absorptometry (DEXA) bone scan performed in 2008 revealed a T-score for the hip of 2.8 and for the lumbar spine of 3.2, consistent with osteoporosis.At that time, he was prescribed analgesics, bisphosphonate, rocaltrol and calcium lactate. He was also prescribed a thoracolumbar brace and advised to undergo regular physiotherapy. Upon follow up, patient claimed that the back pain had improved.

Results of a serial DEXA bone scan 9 months following treatment showed an improved T-score of hip of -1.9 and the lumbar spine of -2.7. Currently, he is still scheduled for orthopaedic follow-up that inludes yearly DEXA bone scan monitoring.

\section{DISCUSSION}

Suprasellar germinoma is a rare intracranial germ cell tumour with variable clinical presentations. Although most 


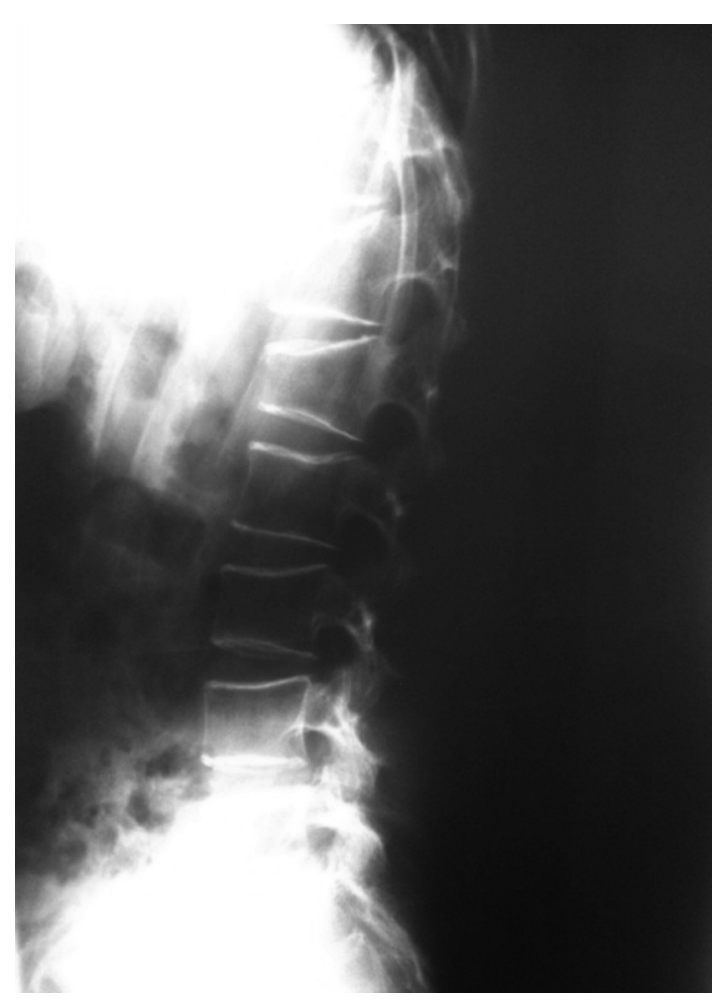

Spine radiographs showed $\mathrm{T} 10, \mathrm{~T} 12$ and $\mathrm{L} 1$ compression fractures with osteopenic changes.

clinical presentations are not related to the field of orthopaedics, we must bear in mind that endocrine complications of panhypopituitarism pose high morbidity risks to the patient and need early attention. Few cases of suprasellar germinoma were reported in literature regarding effects of the brain mass along with an endocrine deficit. Most reports do not mention long term complications arising from panhypopituitarism. One of our concerns should be secondary osteoporosis.

Secondary osteoporosis can arise as a consequence of endocrinopathies. Bone remodelling is also dependent on sex hormones. Estrogen primarily exerts its effect on bone through regulation of osteoclasts which inhibits osteoclast formation and activity while increasing the apoptotic rate of these cells. Much of the action of testosterone occurs as a result of its aromatization to estrogen ${ }^{2}$. Testosterone exerts an additional effect primarily on osteoblasts by increasing proliferation and decreasing apoptosis. Both estrogen and testosterone may affect bone by modulating the responsiveness of osteoblasts to parathyroid hormone ${ }^{3}$. In addition, gonadal hormones affect development of bone morphology during growth. Secondary osteoporosis in these patients is multifactorial, with causes that include endocrinopathy and adverse effects of irradiation.

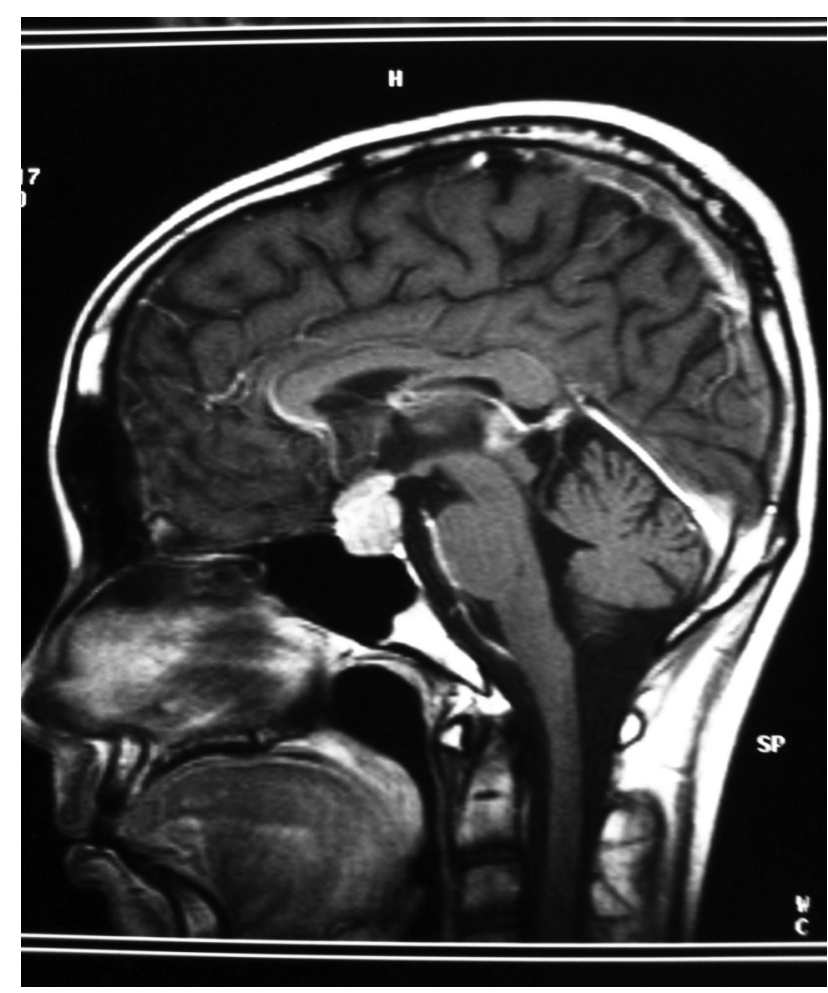

MRI showed suprasellar germinoma

Unfortunately, many patients with osteoporosis are identified only after they have sustained a fracture. The diagnosis of osteoporosis should be established with a DEXA scan. In the case described here, endocrinopathy presented in an adolescent patient resulting in secondary osteoporosis that interfered with development of peak bone mass, and thus increased the risk of future fracture. Yearly DEXA scan should be performed for evaluation of disease progression and the effectiveness of anti-osteoporotic treatment.

Treatment of secondary osteoporosis entails education, instructions for modification of lifestyle factors and treatment of the underlying disease. If complications arise such as vertebral fracture, additional intervention is needed to treat the resultant problem. In adition to treatments mentioned earlier, bisphosphonate has been found to be effective in improving bone mineral density and is targeted towards limiting further bone loss, thus decreasing risk of fractures and its morbidity. Treatment of secondary osteoporosis is more complex than that of primary osteoporosis. There are several studies pertaining the use of bisphosphonate therapy for children with secondary osteoporosis; however bisphosphonate therapy in paediatric and adolescent patients remains controversial because of inadequate long-term efficacy and safety data ${ }^{4}$. For this reason, many experts recommend limiting use of these agents to those children and adolescents with symptomatic 
vertebral collapse, and reduced bone mass ${ }^{4}$. Short term bisphosphonate use appears to be well tolerated,but more data is needed to conclude for the long term effect.

In conclusion, despite hormonal treatment for panhypopituitarism secondary to suprasellar germinoma, incidence of secondary osteoporosis is still noted in many cases. Secondary osteoporosis in such cases may not be detected early and thus remain clinically silent until the patient presents with multiple atraumatic compression fractures. Although osteoporosis in these instances is due to endocrine disorder, hormone replacement is insufficient for prevention of osteoporosis. It is devastating for a young patient to develop secondary osteoporosis with multiple vertebral fractures. To lower the risk of additional fractures and preserve the quality of life in these patients, interventions should be initiated early. Hence, it is important to consider osteoporotic fractures as a complication in a patient with suprasellar germinoma.

\section{REFERENCES}

1. Jennings MT, Gelman R, Hochberg F. Intracranial germ-cell tumors: natural history and pathogenesis. J Neurosurg. 1985; 63(2): 155-67.

2. Riggs BL, Khosla S, Melton LJ III. Sex steroids and the construction and conservation of the adult skeleton. Endocr Rev. 2002; 23: 279-302.

3. Leder BZ, Smith MR, Fallon MA, Lee M-LT, Finkelstein JS. Effects of gonadal steroid suppression on skeletal sensitivity to parathyroid hormone in men. J Clin Endocrinol Metab. 2001; 86: 511-6.

4. Bacharach LK, Ward LM. Clinical Review: bisphosphonate use in childhood osteoporosis, J Clin Endocrinol Metab. 2009; 94(2): 400-9. 